\title{
A study on enabling Deep Learning in IoT for Disaster Management
}

\author{
Aditya Raj, \\ Dept. of Electronics and Communication Engineering, Mar Baselios College of Engineering and Technology, \\ Trivandrum. \\ adityaraj1797@gmail.com
}

\begin{abstract}
With increasing population there is equally higher risk of laosing precious life due to natural calamities like Earthquakes, Floods, landslides etc. every year. We can minimize this risk by sensing the changes in advance and providing advance information with the help of exploding technologies. Internet of Things (IoT) is one such promising technology which has gained attention of researchers due to seamless connectivity between people and object. IoT represents a system where sensors are attached to objects via internet and thus provides real time data monitoring. The sensed data coming from IoT systems are not streamed properly. These data can be analyzed effectively by another promising technology called Deep Learning (DL) that can predict the likely possibility of a disaster threat. With the available information sensed using IoT devices incorporated withDeep Learning(DL) technique, we can predict the occurrence of a disaster looming in and thus minimizing the risk of losing life.
\end{abstract}

Index terms: IoT, Deep Learning, Disaster management.

\section{Introduction}

As the technology is growing in a faster rate, automation is very easier in this era. So many promising technologies are there which decreases the man power without compromising the efficiency. Some among them are Internet of Things (IoT), Deep Learning (DL), Big Data, Assistive technologies, Block Chain, etc. In this work, DL is incorporated with IoT for efficient disaster Management.

In the era of the Internet of Things (IoT), an enormous amount of sensing devices, connected to the network, collect and generate various sensory data, which are of different types, over time for a wide range of fields and applications. Through this technology, one can connect sensor data to outside world in real time and continues monitoring is possible to a great extent. Based on the nature of the application, sensing devices will result in big or fast/real-time data streams.
With the help of the advanced machine learning techniques namely Deep Learning (DL), these huge amount of data can be handled in an easier way. This technology brings two important improvements over the traditional machine learning approaches in the two phases of training and prediction. First, they reduce the need for hand crafted and engineered feature sets to be used for the training. That means, through training the network itself is extracting the features. Consequently, some features that might not be apparent to a human view can be extracted easily by DL. This will facilitate the analytics and learning in IoT domain. Secondly, as the network is doing training by itself, it can predict the output more efficiently and effectively in any of the circumstances if the training data is correct.Only one drawback of this technology is the unavailability of huge amount of data. But in IoT domain, this gets eliminated as the data size is very huge. Second, the trained system can be reused only by changing the final layers for similar types of applications.

As the IoT data is of very large in size, analyzing and processing of this data will be a time consuming process. Also we couldn't extract so many unique feature from the sensed data, if not handled properly and securely. For extracting these features, a powerful network which can handle a huge amount of data with high efficiency within a limited period of time is required. Deep neural networks can be used for the purpose. Nowadays, different types of networks are available which can handle these data in a highly secure manner [1].

Natural disasters may occur abruptly, but nature will always give some sort of warning before the onset of a disaster. If we can monitor it efficiently, we can prevent them to some extent [4] [7]. In this paper, I am proposing a new method for incorporating DL in IoT for efficient real time monitoring purpose. Using this method I'm proposing a technique for predicting disaster and its impacts. The paper is organized as follows. Section 2 explains about IoT followed by section 3 deep learning. Section 4 explains DL in IoT followed by the proposed method in section 5 and conclusion in section 6 . 


\section{Internet of Things}

Internet of Things (IoT) term was first said by Kevin Ashton in 1999. The concept of Internet of Things deals with the use of electronicdevices which can continuously monitor and capture data which are then connected either to private or to public cloud, enabling to automatically trigger certain events. The idea behind this technology deals with device to device communication with devices or with humans, IoT is a platform provider for the objects to sense and to control other devices connected together to form a meaningful solution to real world problem.

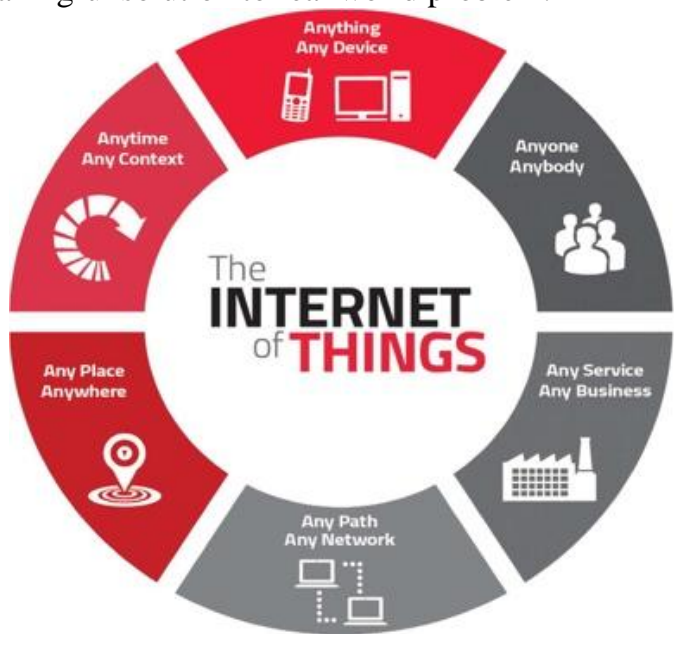

Figure 1: Internet of Things

IoT basically provides connection between different objects (sensors) through a connecting medium which can be either wireless or wired. When object are connected in this fashion, for example sensors, they can sense the parameters and we can perform actions according to the situation. And this is known as smart objects. Basically IoT make day to day life easy.

Consider an IoT system that is continuously monitoring a parameter and sending it to the cloud. Then the amount of data it is sensing is very huge in size. If it is continuously sensing more than one parameter, then within seconds the amount of data generated is very large in size and we can't be able to distinguish which sensor is sensing that data, at what time these data get generated, from which sensor is it coming, like that so many queries arises and we need to find the correct solution. This may not be possible always as the generated data size is very large. This data can be analyzed by Deep Learning techniques.

\section{DEEP LEARNING}

"Artificial intelligence (AI) is the ability of a machine which can replicate the intelligent human behaviour. AI is obtained by a imitating intelligent human behaviour like thinking, learning and decision making when trying to solve a problem. Artificial Intelligence have various subfields. "Machine learning is a sub-field of AI" which uses different algorithms for doing a specific tasks. "Deep learning is a sub-field of Machine Learning" where a big data can be handled in the most effective way where machine learning fails.

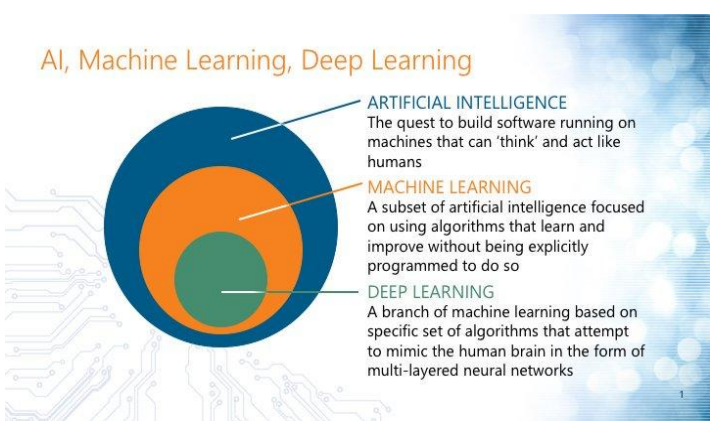

Figure 2: Subsets of Artificial Intelligence

\subsection{Machine Learning (ML)}

Machine learning is an AI subset which allows computers to learn without being programmed explicitly. Machine is trained on a huge amount of data to create a deep learning model that helps to arrive at conclusions from the learned features. So the machine is trained in such away as to identify unique features from the input dataset, for forming the feature vector and creating a model for the unique identification of a particular object.

Deep Learning (DL) is a subset of Machine Learning based on some specific set of algorithms which try to mimic human neural system using multilayered neural network. In order to extract the right and unique feature on its own, DL networks need high dimensionality data. DL network will identify and extract the unique features from the given dataset. Therefore the number of inputs required is very large. Since in DL network the amount of the data required is large, dimensionality of the Feature vector cannot be predicted.

\section{Example of Machine Learning}

1. At first, we have to collect a dataset containing different flowers along with their respective species. Then unique features of each species are 
to be identified and extracted form feature vectors using the extracted features and these features are acting as input to the network.

2. Design a network suitable for that particular feature vectors. The network complexity depends on the dimension of the feature vector.

3. The formed feature vectors which contains various characteristics of different species of flowers will be fed to the network. Using this, the machine will be trained and create a model which can be used to classify flowers into different categories.
4. After training, the model can be tested by giving another set of inputs that feature set should also be in the same format as that of the trained inputs that means the testing should be done with the feature vector having the same unique features.

Then this model will output the species of the flower present in the new input data set. This process of training a machine to create a model and use it for decision making is called Machine Learning. However this process has some limitations

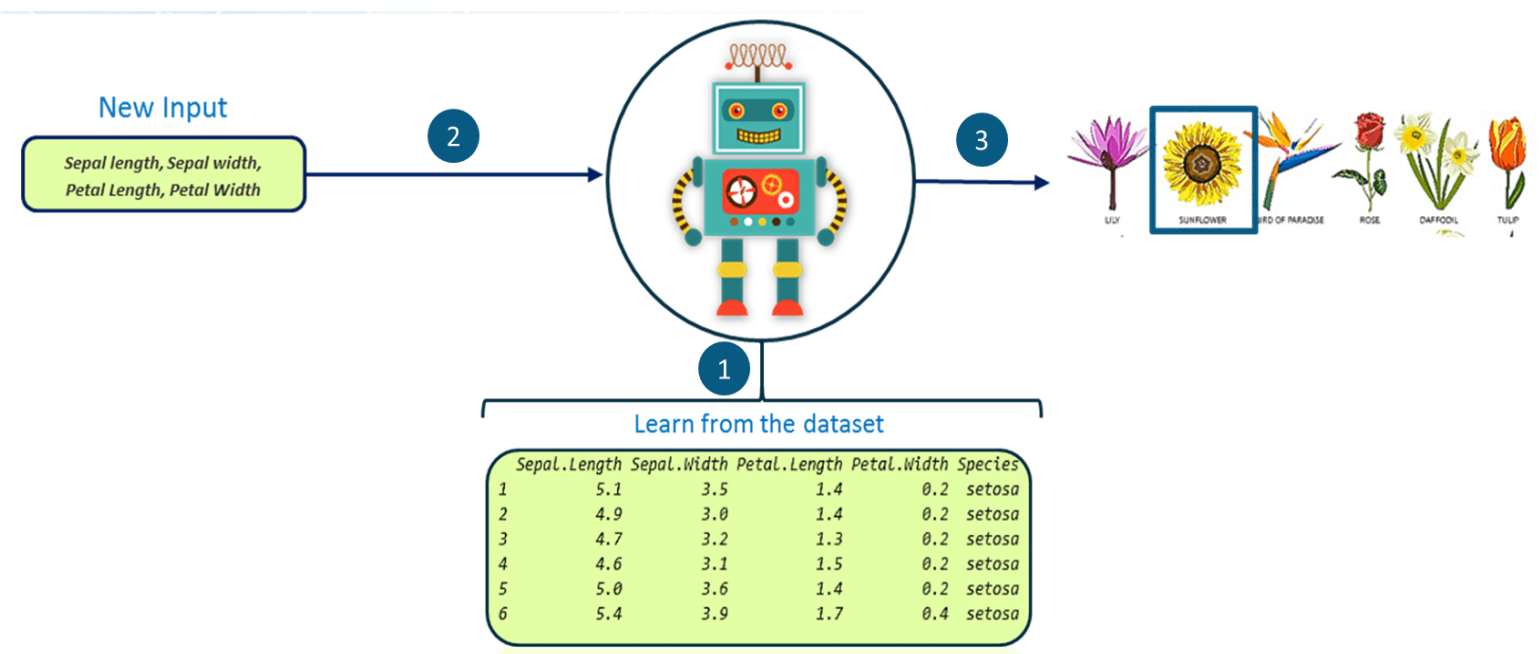

Figure 3: Example to show Machine Learning

\section{Limitations of Machine Learning}

1. ML cannot solve crucial AI problems that contains enormous amount of highly varying data's like Natural language processing, Image recognition etc. because in these cases we couldn't fix the dimensionality of the input data vector.

2. With ML, feature grouping and mapping may not be correct in the current scenario. That means, there is no efficient algorithm is existing for efficient grouping or mapping of features.

3. In the above said example, if an input comes with different pose, machine may not identify the flower correctly because there may be some other unique feature that can be used for identifying that particular pose. In order to make the machine correctly identify this, we have to retrain the machine with another feature vector indicating that particular pose. Thus dimensionality of the feature vector increases. But ML is not useful while working with high dimensional data.
To overcome these limitations, a new technology was introduced in 2006, which came to practice in 2010 that is known as Deep Learning. This technology is also using the same neural network architecture, but in a different manner.

\subsection{Deep Neural Networks}

The brain consists of several connected neurons known as the neural network. A neural network's basic building block is known as a perceptron. Artificial neurons are a deep neural network. All Deep Neural Networks consist of three different stacks of layers: Input Layer, Hidden Layer and Output Layer

The input layer receives all the inputs the hidden layer extracts unique features and the output layer gives the conclusion The number of neurons in the input layer depends on the size of the input data and on the specific application in the output layer. All layers are termed hidden layers in between input and output layer. Each hidden layer in DL network comprises of a stack of certain layers desired for some particular task. The number of hidden layers in the hidden layer stack depends on the complexity of the input data and the application 
to be used. In deep learning, the number of hidden layers can be increased for doing different functions. As the network is learning itself by extracting uniqueness from the input data, the number on hidden layers required will be very high compared to an ordinary ML neural network. But as the hidden layer increases the network complexity will increase, but the accuracy will also increase.

Different Deep Learning networks are available in literature. Some of them are given in table I. From the above mentioned DL networks for Brain Tumour detection from MRI, CNN (convolutional Neural Network ) is the best. data. The hidden layers can be broadly classified as three different types according to the features they are extracting. First type will extract low level features, second will extract mid-level features and last type will extract high level features. If we have 2 sets of data that are similar in some sense and if we want to create 2 different networks, then we can create one for the first dataset and reuse the first 2 types of hidden layers for creating the second dataset. That means, we need to retrain only the third type of hidden layers for constructing the full network as the first two types are already trained with a similar dataset. Thus network reusability is also possible.

\section{Simple Neural Network}

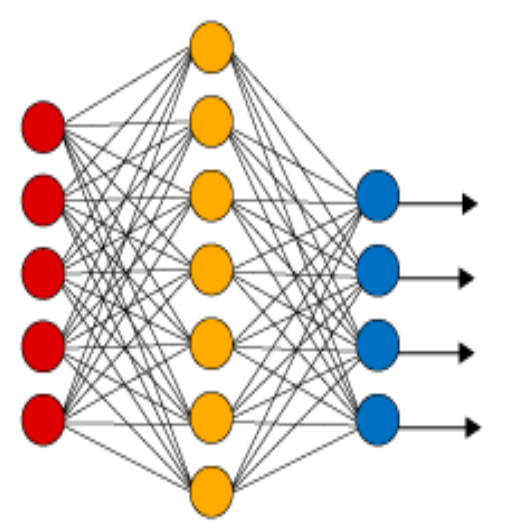

Input Layer

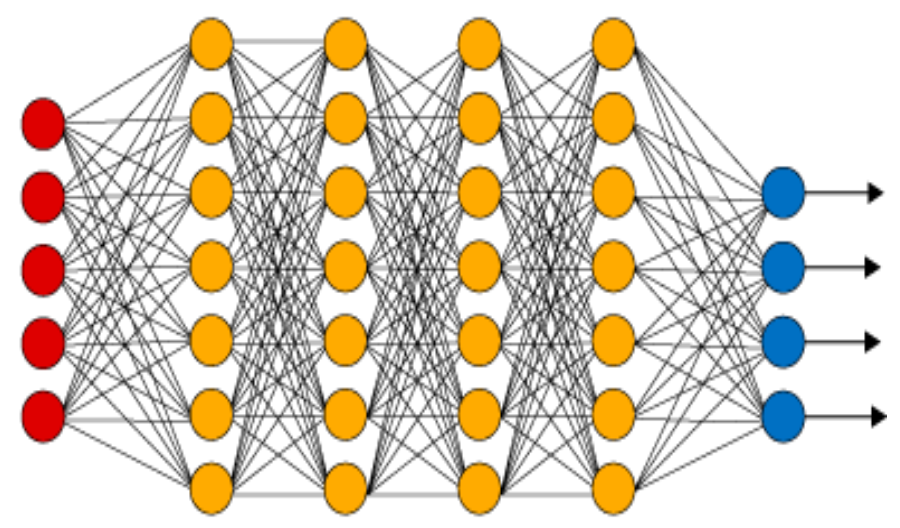

Hidden Layer

Figure 4: Deep Neural Network

\subsection{CNN (Convolutional Neural Network)}

Deep CNN consist of several layers which can process large amount of data using linear as well as non-linear operators that are learned simultaneously to solve a particular problem. In other words, it is a $3 \mathrm{~d}$ collection of nodes that form several layers that process internally and transform an input to produce an output. Like ordinary neural networks made of neurons with learning weights and biases. CNN's main parts are

1. Convolutional layer

2. Rectified Linear Unit (ReLU)

3. Pooling layer

A stack of these 3 layers are repeated several times periodically to from the Deep CNNs.

Convolution layer is performing convolution operation using a set of learnable filters to extract unique features from the input data that can be used as the input for the upcoming layers in a different manner. The selection and formation of learnable filters depends on the application. Every filter or kernel is of one-dimension or two-dimension, depends upon the dimension of the input data and can be small in size but can be extended through the full depth of the input volume. Normally square kernels are used if the input data is a $2 \mathrm{D}$ or a $3 \mathrm{D}$ one. As the convolutional layer is performing convolution operation, the network is not fully connected and thus it decreases the network complexity. In order to understand the $\mathrm{CNN}$, we need to know the 3 key concepts.

\section{Local Repetitive Fields}

In the typical neural network, all neurons in the in input layer are connected to neurons in the first hidden layer, but in $\mathrm{CNN}$, the some neurons in the input layer are connected to the neurons in the hidden layer in small regions of the kernel size. These are called local repetitive fields and are translated from the input layer to the hidden layer through an image to create a feature map. This will reduce the dimension of the parameter space. 


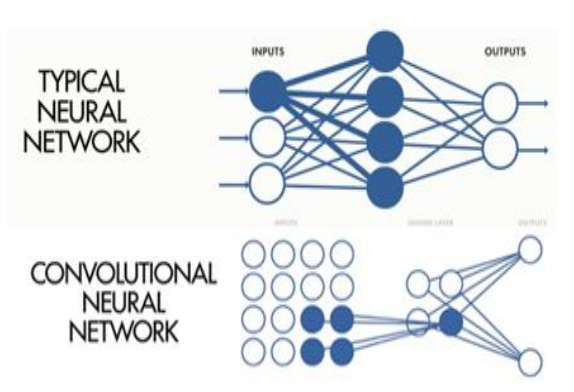

Figure 5: Basic Difference between Typical Neural Network and CNN

\section{Shared Weights and Bias}

The weights and biases are known as the trainable filter parameters. Typical neural network have neuron with weight and bias values as parameters, but the value of these parameters changes if there is mismatch in the outputs to make the relation: "Desired output equal to the Actual output". In convolution layer, the weight and bias can be made same for all the neurons in a particular stack of the hidden layers. That means all neurons in particular layer will detect the same features to identify the object correctly.

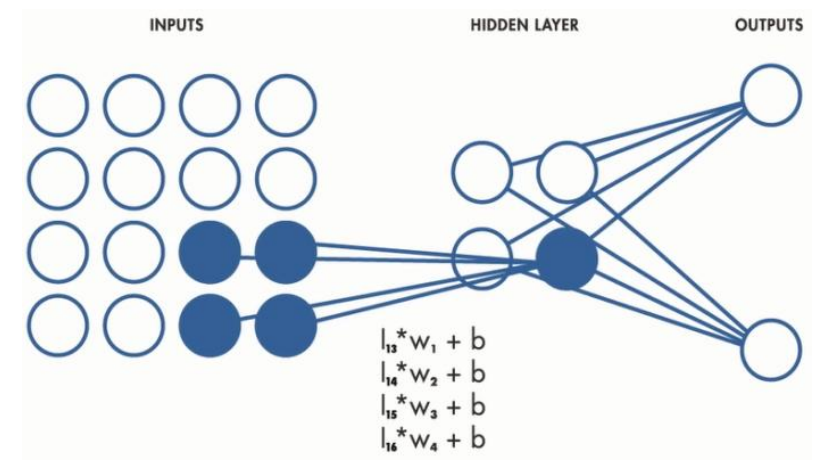

Figure 6: Shared Weights and Bias

\section{Activation and Pooling}

In the activation step, the activation function applies a transformation to the output of preceding neuron. The most commonly used activation function in $\mathrm{CNN}$ is the rectified linear unit (ReLU). This function works in such a way that if the input is a positive value, the same value is mapped and if the input is a negative value, it is mapped to zero as shown in the figure.

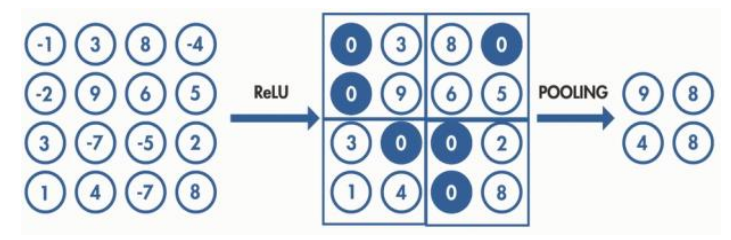

Figure 7: Activation and Pooling

\section{Deep learning in IoT}

IoT is about devices, data and connectivity. The real value of Internet of Things is about creating smarter products using variable sensors, delivering intelligent insights into the real world problems and solutions. As millions of devices get connected to internet, IoT will trigger a massive inflow of huge amount of data, the quantity of that cannot be predicted [2]. The key challenge is visualizing and uncovering insights from various types of data that can be divided into structured, unstructured, images, contextual, dark data, real-time. Structured data can be streamed and analyzed easily whereas others can't. Identifying a particular data that has a specific property from a huge amount of data is a time consuming task. Using Deep Learning technology we can do it with less time and more efficiently. This technology is the key that enables for smarter devices and a connected world [3].

Deep learning technology can be used as a key differentiator to derive insights rapidly from massive streams of IoT data. With IoT one can monitor and analyze real time data and this would help in real-time decision making, object detecting, instance detecting, etc.

\section{Proposed Method}

Natural disasters are common and it is caused by climatic changes, weather conditions, environment imbalance due to pollution, etc. and will represent a significant risk to the human life, community and social life [4]. If we can find a solution for predicting different types of natural disasters, then we can save precious human life. Through this paper new and efficient method is proposed which incorporates DL in IoT for disaster prediction. The block schematic of the proposed method is shown in fig 8 .

At first the sensors will continuously monitors and the sensed data will go to the DL model. Types of sensors used vary depends upon the application to be used.

Sensed data will move to the DL section. DL section comprises of a model which can be a onedimensional or a two dimensional model depends upon the input data. In this, we need to classify the sensed data into different classes such as if it corresponds to some disaster or not. So here, a classification network is to be used. 
DL network has 2 phases: a training phase and a testing phase. At first the network is to be trained using the training dataset. The training dataset can be taken from the sensed data output. But the data that causes disaster are difficult to sense naturally. Therefore, sufficient amount of data, in all circumstances, may not be available. If so, data for training and testing can be created by another DL network called as Generative Adversarial Network (GAN). After training phase the sensed data is fed to the network as test data and DL network evaluates and predicts the result depending on the training. The DL output is send to cloud and the cloud will take action according to this result.

Consider an example where the sensed data, which is the water level information, is coming from the reservoir. In this case, the DL section should be trained in such a way that the end result comprises of one of the 3 categories: normal, low risk, and high risk. This information is fed to the cloud. Then the cloud will take decisions as follows.

1. If normal, the cloud system will not send any notifications.

2. If low risk, then notifications will be send to authorities and nearby people about the water level in which they can viewed through an app.

3. If the result falls on to High risk Category the notifications will be send to the police so that they can take necessary immediate actions, next to the hospital's so that they can take precautions, then to the people leaving there for immediate evacuation and to authorities to take necessary steps to prevent it.

\begin{tabular}{|c|c|c|}
\hline Application & Sensors & Uses \\
\hline \multirow{2}{*}{ Earthquakes } & Accelerometers & $\begin{array}{l}\text { To measure large amplitude and high frequency seismic } \\
\text { waves }\end{array}$ \\
\hline & Seismic wave sensor & $\begin{array}{l}\text { To measure seismic vibrations by converting ground } \\
\text { motion into a measurable electronic signal }\end{array}$ \\
\hline \multirow[b]{2}{*}{ Flood } & Water level sensor & To sense the water levels of the reservoir \\
\hline & CCTV Cameras & $\begin{array}{l}\text { To capture the images of water levels which can be given } \\
\text { to the DL model as the test data }\end{array}$ \\
\hline \multirow{5}{*}{ Landslides } & Tilt sensor & $\begin{array}{l}\text { To detect the direction and inclination of a reference } \\
\text { plane }\end{array}$ \\
\hline & Moisture Sensor & Tomeasure the moisture content in the soil \\
\hline & Strain Gauge & $\begin{array}{l}\text { Tomeasure the ground displacements due to natural } \\
\text { calamities }\end{array}$ \\
\hline & Pressure sensor & $\begin{array}{l}\text { Tomeasure the pressure of the air in a small enclosure } \\
\text { that is air in the compressed form }\end{array}$ \\
\hline & Geophone & To convert ground movement (velocity) into voltage \\
\hline
\end{tabular}

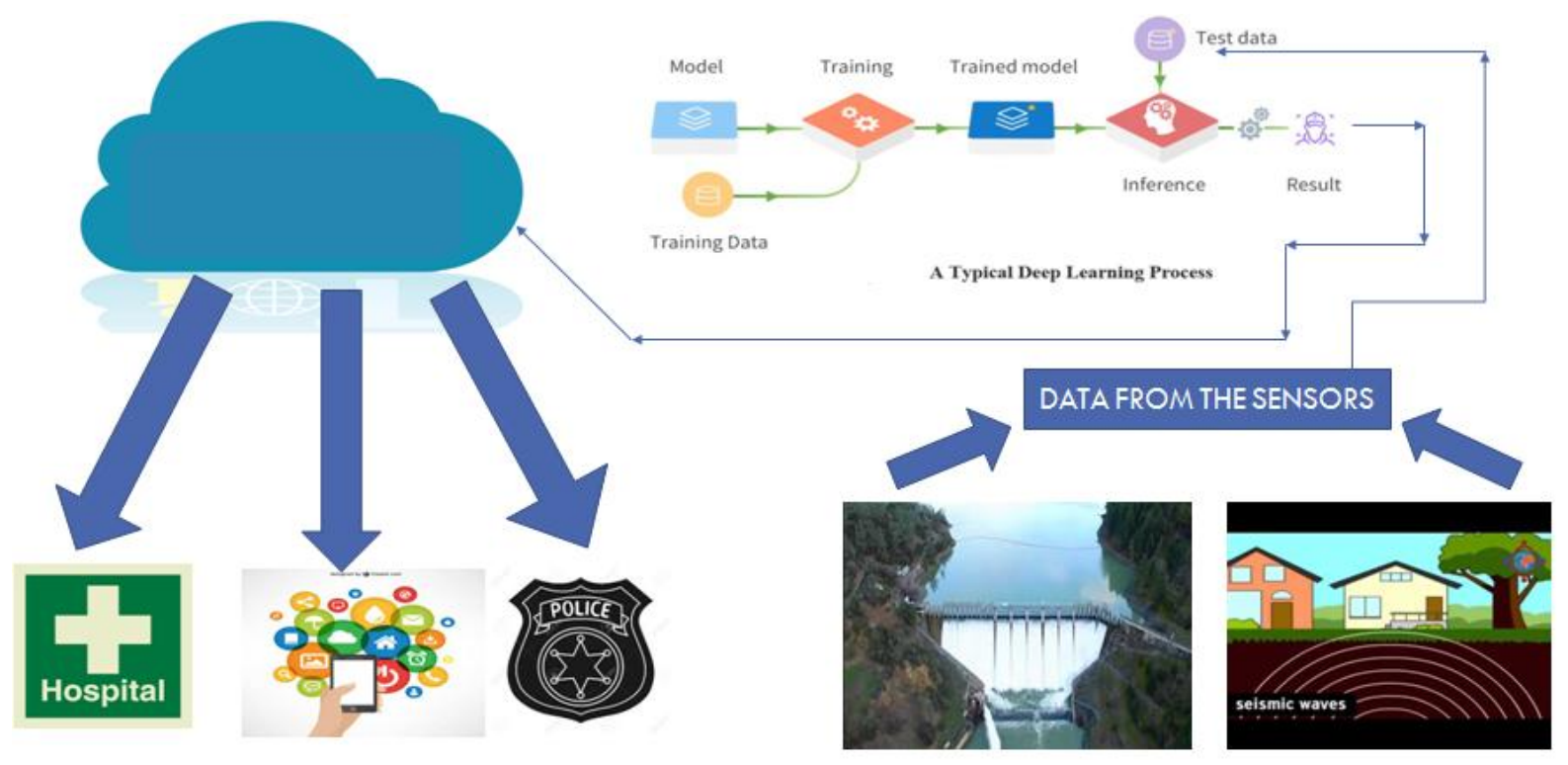

Fig 8: Block diagram of the proposed method 
In the above said example, if we are using CCTV cameras, then the sensed data will be images and thereby we need to adopt a 2D-DL model.

Through this paper, I have proposed a method for analysing and preventing disasters by incorporating 2 technologies IoT and DL. With the available information I have gone through, this one initiative can be a game changer to prevent one more disaster and thus save precious lives.

\section{Conclusion}

Deep Learning and Internet of Things have drawn the peoples attention in recent years, as these two technology made a vibrant and positive impact in peoples life. IoT and DL can be considered as chain in which IoT produces large amount of data and it is analyzed by DL . DL models produce highlevel abstraction and insight that is fed to the IoT systems for fine-tuning and improvement of services.

In future, this can be implemented using one of the one-dimensional Deep Learning models known as Long Short Term Memory (LSTM). This is a model extended from Recurrent Neural Network (RNN). The same methodology can be extended for predicting other natural disasters too.

\section{References}

[1] Mehdi Mohammadi, Ala Al-Fuqaha, SamehSorour and Mohsen Guizani, "Deep Learning for IoT Big Data and Streaming Analytics: A Survey", IEEE Journal on Communication Surveys and Tutorials (COMS\&T), pp 1-40, June 2018.

[2] Jie Tang, Dawei Sun, Shaoshan Liu and JeanLuc Gaudiot, "Enabling Deep Learning on IoT Devices", IEEE Computer Journals, Vol. 50, issue 10, pp 92-96, October 2017.

[3] Shuochao Yao, Yiran Zhao, Aston Zhang, Shaohan Hu, Huajie Shao, Chao Zhang, Lu Su and Tarek Abdelzaher, "Deep Learning for the Internet of Things", IEEE Computer Journals, Vol. 51, issue 5, pp 32-41, March 2018.

[4] ParthaPratim Ray, Mithun Mukherjee, (Member, IEEE), and Lei Shu (Senior Member, IEEE), "Internet of Things for Disaster Management: State-of-the-Art and Prospects", IEEE Access, September 14, 2017.

[5] https://www.edureka.co/blog/deep-learningtutorial?utm_source=youtube\&utm_campaign=dee p-learning-180717 wr\&utm_medium=description
[6] https://www.mathworks.com/campaigns/offers /deep-learning.html?s_eid=PSM_15028

[7] https://azure.microsoft.com/es-es/blog/using-aiand-iot-for-disaster-management 